\title{
Expérience professionnelle : capital ou fardeau pour les seniors?
}

Professional Experience: a real Capital for older Workers?

\section{Stéphane Bellini}

\section{(2) OpenEdition}

1 Journals

Édition électronique

URL : http://journals.openedition.org/travailemploi/3784

DOI : 10.4000/travailemploi.3784

ISSN : 1775-416X

Éditeur

DARES - Ministère du Travail

\section{Édition imprimée}

Date de publication : 15 mars 2007

Pagination : 81-89

ISBN : ISSN : 0224-4365

ISSN : 0224-4365

\section{Référence électronique}

Stéphane Bellini, «Expérience professionnelle : capital ou fardeau pour les seniors? 》, Travail et Emploi [En ligne], 109 | janvier-mars 2007, mis en ligne le 15 mars 2009, consulté le 30 avril 2019. URL : http:// journals.openedition.org/travailemploi/3784 ; DOI : 10.4000/travailemploi.3784 


\title{
L'expérience professionnelle: capital ou fardeau pour les seniors?
}

\author{
Stéphane BELLINI (*)
}

L'expérience des seniors est loin d'être reconnue comme leur principal capital par les entreprises. L'auteur constate au contraire la faible ampleur des dispositifs concernant le transfert des compétences acquises par l'expérience. Des arguments rationnels, comme l'évolution des technologies ou le développement de la polycompétence, vont légitimer cette faible prise en compte de l'expérience des seniors. Mais ils ne l'expliquent que partiellement. À côté de la dévalorisation de leurs savoirs techniques et codifiés, l'article met en évidence l'absence de visibilité dont pâtissent leurs savoirs tacites, souvent attachés non pas à tel ou tel individu mais à des collectifs de salariés et à la relation individu-organisation. C'est la notion même d'expérience qui est questionnée. En la réduisant à la somme des savoir-faire techniques acquis dans l'emploi, on ne prend pas en compte la compétence professionnelle acquise par l'analyse réflexive et le questionnement sur son travail qu'opère le salarié. À défaut de pouvoir être valorisée financièrement, l'expérience n'a finalement qu'un coût croissant avec l'ancienneté. De ces représentations, il résulte un risque d'éviction rationnelle des seniors très difficile à contrebattre. L'article conclut à l'utilité de la reconnaissance des acquis de l'expérience pour tous, dans toutes ses dimensions.

L'expérience professionnelle est couramment perçue comme un atout pour les salariés âgés, souvent qualifiés de seniors. Pourtant, les actions de GRH des entreprises indiquent qu'elle est concrètement peu prise en compte. Lors d'une précédente enquête sur les pratiques de gestion des seniors, nous avions relevé un certain nombre de pratiques de transfert de compétences acquises par l'expérience (par exemple conservation de la mémoire des gestes au travail ou tutorat). Des actions ponctuelles sont mises en œuvre, décidées au cas par cas, pour quelques-uns et/ou en réaction à des événements particuliers, mais on ne peut parler de politiques de transfert de compétences, c'est-à-dire d'ensembles d'actions coordonnées vers un objectif de moyen terme. Les politiques de gestion des seniors les plus fréquentes sont marquées par l'organisation de départs anticipés (Bellini, Duyck, Laval, Renaud, VAUCLIN, 2006), c'est-à-dire qu'elles visent à se séparer de salariés qui bénéficient d'une forte expérience professionnelle. Nous nous interrogeons ici sur les raisons qui peuvent expliquer ces constats: alors que les salariés âgés sont réputés disposer d'une forte expérience, pourquoi celle-ci n'est-elle pas valorisée? Est-ce à dire que les bienfaits de l'expérience des seniors sont jugés peu utiles?

(*) IUT de Poitiers, département GEA, membre du CEREGE (Centre de recherche en gestion), laboratoire de l'IAE de Poitiers, email : stephane.bellini@univ-poitiers.fr
Après avoir montré que l'expérience professionnelle est loin de constituer systématiquement un atout pour les salariés âgés, nous cherchons à identifier les raisons de sa faible valorisation. Cela nous conduit à nous interroger sur le processus d'évaluation de l'expérience. Cette recherche s'inscrit au cœur des réflexions sur le maintien dans l'emploi des seniors, dont les incitations européennes à développer leur emploi en France (accords de Lisbonne) et le contrat «senior» montrent l'actualité ( $c f$. encadré 1).

\section{L'expérience professionnelle des seniors: un capital remis en cause}

Il est habituel de considérer l'expérience des anciens comme leur principal capital. Cette acception peut même être considérée comme «triviale», selon l'expression d'un des DRH rencontrés, tant «le discours sur l'expérience est, de façon récurrente, un discours de succès» (AstiER, 2004, p. 34).

Pourtant, qu'est-il fait de ce joyau que constituerait l'expérience? Comment est-elle valorisée, prise en compte dans les dispositifs de transmission des compétences? Comme d'autres chercheurs, nous constatons la très faible ampleur des dispositifs concernant le transfert de compétences acquises par l'expérience (BELlini et alii, ibid.; MinNI, Topiol, 2002 ; FALCOZ, 2004). MinNi et Topiol (2004) consta- 


\section{Encadré 1}

\section{Dispositif méthodologique}

Pour ce travail, nous avons procédé à une enquête auprès de quinze entreprises de la région Poitou-Charentes, inscrite dans une demande de l'ARACT (1) Poitou-Charentes et plus largement dans le cadre d'un projet européen «À tout Âge». Visant initialement à identifier sur la région les «bonnes» pratiques de gestion des âges, la problématique a été élargie non seulement pour les recenser, mais aussi les comprendre en appréhendant leurs contextes (social, technologique, économique...). Pour ce faire, nous avons eu recours à des entretiens semi-directifs avec le DRH de chaque organisation, parfois complétés d'entretiens avec le personnel de médecine du travail. L'objectif est de procéder au recueil des données in situ dans leur «cadre naturel » (GIODARNo, 2003, p. 16). La durée des entretiens a oscillé entre 1 heure 20 et 2 heures. Le guide d'entretien cherche à recueillir des informations sur les thèmes suivants:

- l'activité et les contextes de l'entreprise (technologique, concurrentiel, financier, historique, stratégique, social); - les caractéristiques du travail: organisation concrète, horaires, types de difficulté (pénibilité physique, pression psychologique, sollicitations cognitives), compétences requises, changements technologiques introduits;

- les indicateurs sociaux: AT, MP, absentéisme, "gestion» de l'inaptitude, niveaux de qualification, turn-over, demandes du CHSCT, etc.

- les pratiques de GRH (orientation du plan de formation, mode de recrutement, priorités du DRH), et plus précisément les pratiques:

-spécifiques aux seniors (plus de 50 ans), qu'elles soient formalisées ou non, en notant l'ampleur des pratiques (anecdotiques vs systématiques);

- particulières comme le mode de transfert des compétences aux plus jeunes ou l'utilisation des nouvelles technologies pour pallier les carences de l'usure au travail.

Le guide est orienté vers l'identification des pratiques de gestion dans leurs contextes. II n'est pas directement centré sur l'expérience professionnelle. Cependant, la notion est à chaque fois évoquée à travers les actions destinées aux seniors. De plus, en ancrant les entretiens sur des pratiques, des faits et non un discours, nous recueillons une information qui nous éclaire sur les usages concrets de l'expérience plus que sur l'opinion sur celle-ci.

L'exploitation des entretiens s'est effectuée sur la base d'une grille classant les items selon la trame du guide d'entretien.

\section{Choix des entreprises}

Le choix des entreprises a suivi deux critères. II s'est d'abord agi de respecter, dans toute la mesure du possible, la variété des secteurs de l'économie régionale. Nous avons ensuite fait le choix de contacter les entreprises disposant de $\mathrm{DRH}$, car ces derniers sont chargés de concevoir et/ou mettre en œuvre les pratiques de gestion des seniors que nous souhaitions identifier au départ. Le choix du DRH comme interlocuteur signifie que toute généralisation de nos constats aux TPE et PME de tailles modestes est exclue de fait; cela constitue une des limites de ce travail

Le tableau ci-dessous présente les principales informations sur les sociétés visitées. Pour des raisons de confidentialité, le nom réel des entreprises a été dissimulé sous un pseudonyme.

Tableau 1

Les entreprises étudiées

\begin{tabular}{|l|l|c|}
\hline \multicolumn{1}{|c|}{ Entreprise } & \multicolumn{1}{|c|}{ Activité } & Nombre de salariés site \\
\hline Flaco & Verrerie & 430 \\
\hline Char & Production de chariots élévateurs & 600 \\
\hline Cendrillon & Carosserie pour poids lourds & 150 \\
\hline Satelinergie & Production de batteries pour satellites & 620 \\
\hline Flying System & Maintenance d'instruments de navigation aérienne & 420 \\
\hline Schtak & Abattoir et transformation de viande porcine et bovine & 470 \\
\hline Davia & Aviation & 162 \\
\hline Aquatis & Appareils destinés à la filtration d'eau & 120 \\
\hline Electronis & Appareils électromécaniques et électroniques & 455 \\
\hline Territoria & Pilotage et réalisation d'opérations d'aménagement du territoire & 700 \\
\hline Prevoir & Compagnie d'assurance mutualiste & 1600 \\
\hline Royal & Fabrication de bottes et vêtements de plein air & 670 \\
\hline Maisonbois & Fabrication de meubles de cuisine et de salle de bains en bois & 260 \\
\hline Pneumatix & Manufacture de pneumatiques & 540 \\
\hline Electra & Distributeur d'énergie (électricité et gaz) & 300 \\
\hline
\end{tabular}




\section{Champ géographique}

Le travail d'enquête a été effectué dans la région Poitou-Charentes. Les entreprises qui ont fait l'objet d'investigation couvrent le territoire régional. Les investigations ont été menées dans une région où la répartition des emplois par tranches d'âge et par catégories socioprofessionnelles est représentative des statistiques nationales. Cette similitude est confortée lorsque l'on compare la répartition des actifs occupés par grand secteur d'activité (2).

Sur un plan conjoncturel, le marché de l'emploi du Poitou-Charentes suit la tendance de la France métropolitaine aussi bien sur le taux de chômage que sur l'évolution de l'emploi salarié marchand non agricole entre 1997 et 2004, mais aussi sur le plan de la démographie des entreprises. En la matière, la région Poitou-Charentes suit assez fidèlement les tendances nationales.

(1) Association régionale pour l'amélioration des conditions de travail. L'ARACT Poitou-Charentes est une agence du réseau de l'Association nationale pour amélioration des conditions de travail.

(2) Sources INSEE.

tent que les mesures telles que le développement de missions de tutorat impliquant un senior et un jeune pour la transmission de la connaissance, la mise en place de bilans de compétences, le recours à la formation professionnelle pour maintenir le niveau de qualification des plus âgés, ou bien encore des aménagements spécifiques du poste ou du temps de travail, restent le faitd'uneminoritéd'établissements. Selon cette étude, la gestion des âges se résume le plus souvent à l'établissement d'une pyramide des âges. Rappelons également que les seules politiques de gestion des seniors constatées lors de notre étude visent à faciliter les départs des seniors de leurs entreprises, ce que nous pouvons croiser sur le plan national avec les politiques de préretraite. Cellesci n'ont pas été freinées par la peur d'une perte de compétences, le faible taux d'emploi des seniors en témoigne(1). Un ensemble de données concourt à indiquer que l'expérience acquise dans le travail est faiblement prise en compte. Nous nous centrons ici sur la compréhension d'un tel phénomène.

\section{L'ancienneté n'est pas un facteur d'accroissement du savoir-faire}

Durant les entretiens, nos interlocuteurs sont nombreux à affirmer que la détention d'un savoirfaire n'est pas une question d'ancienneté. Ainsi le responsable RH de Cendrillon présente le cas d'un soudeur jeune embauché dans l'entreprise, qui a rapidement compris les consignes et acquis le savoirfaire. Plus encore, il trouve des astuces que les plus anciens n'ont pas, à force de répéter les mêmes tâches sans questionner leurs gestes. Chez Aquatis, Char, Schtak, le cas se répète, ce qui confirme les propos de VINCENS (2001), pour qui l'expérience n'est pas un «produit-joint de l'activité» (p. 22). Avoir une expérience de vingt ans révèle ainsi peu d'information sur l'expérience acquise dans le travail. Ce constat répété questionne la notion d'ex-

(1) Selon EuroStat, en France, le taux d'emploi des 55-64 ans est de $38 \%$ en novembre 2006, soit un des faibles d'Europe. périence professionnelle, notion brouillée par le langage courant (une expérience de trois ans, une première expérience...) et la proximité de celles de compétences, qualifications et savoirs (GRASSER, Rose, 2001).

De plus, la notion d'expérience renvoie à plusieurs niveaux d'analyse : «La notion d'expérience désigne à la fois un engagement dans l'action professionnelle dont on valorise alors les aspects inédits pour le sujet ("faire une expérience"), le "vécu" comme éprouvé de cette action singulière et de ses effets ("vivre une expérience") et la transformation de soi résultant de cet engagement ("avoir une expérience")» (ASTIER, 2004, p. 34). En confondant en un même terme trois processus, deux dimensions (objective à travers les tâches exercées et subjective à travers le vécu de l'activité), l'expérience «se signale par une opacité qui pourrait la faire regarder comme définitivement énigmatique» (Diallo, Clot, 2003, p. 203).

Même si expérience, compétences et savoirs sont des notions fortement imbriquées, une clarification s'impose. Nous nous appuierons pour ce faire sur la terminologie d'AstiER (ibid.) Une expérience constitue un engagement dans l'action, qui permet une confrontation de l'individu, avec ses qualifications et ses savoirs, à une situation professionnelle (Schwartz, 2004; Vincens, 2001; Grasser, Rose, 2000). Chaque expérience procure ainsi des occasions de mettre des savoirs en action, c'est-à-dire d'acquérir des compétences, tacites ou formalisées. La richesse des compétences acquises par l'expérience dépend de la transformation de soi opérée par l'individu à l'occasion de ses expériences. En fonction de son degré de réflexivité, c'est-à-dire de questionnement sur son travail, ses procédures et sur les finalités de l'action, l'individu pourra tirer parti de ses expériences. Ce n'est donc pas le temps passé dans un poste qui ferait la richesse d'une expérience mais l'analyse réflexive que le salarié en produit. L'équation simple selon laquelle les acquis de l'expérience se développeraient mécaniquement avec l'ancienneté demande donc à être pondérée. 


\section{L'expérience des technologies dépassées périme les compétences.}

Dans un certain nombre d'entreprises, les métiers et technologies évoluent. Les salariés les plus anciens n'ont pas été nécessairement formés aux technologies plus récentes, qui prennent progressivement le pas sur les plus anciennes. Prenons le cas d'Electronis. L'entreprise fabrique des appareils électromécaniques et électroniques (claviers et voyants spécifiques destinés aux professionnels - industrie notamment -, colonnes balisées, barrières immatérielles). La fabrication d'appareils électromécaniques décline au profit de l'appareillage électronique, disposant d'une plus forte valeur ajoutée. Pour cette dernière catégorie d'appareils, les jeunes diplômés ont les qualifications nécessaires à leur réalisation. Les plus anciens, longtemps restés sur la fabrication de produits électromécaniques, voient leur activité largement diminuer. À terme, elle risque de disparaître sur le site et d'être fabriquée dans des pays où les coûts salariaux sont moindres. Le DRH ne remplace pas les départs en retraite et lance un plan de formation qui permettrait aux salariés anciens de basculer vers l'activité électronique plus porteuse. Les plus jeunes seraient alors en situation de transfert de compétences vers les plus anciens. En effet, qualifiés pour travailler sur les postes d'appareillage électronique, ils y ont acquis des compétences que les anciens n'ont pas.

Royal vit à peu près la même situation. L'entreprise produisait au départ des bottes en caoutchouc. Depuis une dizaine d'années, constatant le déclin de ce marché, elle s'est lancée dans les vêtements de plein air, pour lesquels l'activité est logistique et commerciale mais absolument pas productive (la production se fait au Maghreb et en Asie). La production et le chiffre d'affaires liés aux bottes diminuent régulièrement tandis que l'activité textile croît. L'entreprise devient de plus en plus distributrice et beaucoup moins productive.

Dans ces deux cas, on le voit, les compétences acquises au cours de l'expérience ne constituent plus un capital personnel quand l'activité qui a permis leur émergence n'existe plus ou décline sévèrement. À certains égards, on pourrait plutôt considérer l'ancienneté comme un obstacle à l'employabilité. À l'image des entreprises citées, les qualifications anciennes ont tendance à enfermer les salariés qui les détiennent dans des activités ellesmêmes anciennes. Dans le secteur informatique, on parle de facteur déqualifiant du temps. Sans pouvoir se confronter à des situations d'apprentissage, les salariés concernés développent des «incapacités acquises» (LinHART, 1991), processus que le temps qui passe rend de plus en plus difficile à inverser car ce sont des gestes, des attitudes et des réflexes de travail incorporés qu'il s'agit de faire évoluer.

Ce problème est crucial dans une économie en mutation. Les plans de sauvegarde de l'emploi révèlent cruellement que l'expérience accumulée dans un même métier, surtout industriel, peut être un obstacle à une reconversion dans d'autres emplois (voir Le Monde Initiatives, mai 2003). Le problème se pose également au sein du secteur tertiaire. Le DRH de la mutuelle Prévoir cite l'opinion des seniors, sondés sur les jeunes: les salariés anciens estiment que les jeunes sont plus armés pour faire face à l'accentuation de la compétition économique. Pour le DRH, l'explication réside dans le changement de méthode de travail. Le type de relation commerciale et les méthodes de travail des seniors seraient l'héritage d'une période où la concurrence était moins vive et où le fonctionnement était plus autocentré que tourné vers le client.

La valorisation de l'expérience des salariés âgés est ici remise en cause. Martin de Holan et PhiLIPS (2004) le confirment en affirmant que « savoir oublier est parfois une nécessité organisationnelle dès lors que l'on doit changer de logique dominante», et particulièrement dans les situations inhabituelles, pour lesquelles aucune solution n'a été répertoriée.

En la matière, Flying System (maintenance d'appareils de navigation aérienne) fait figure d'exception. Comme chez Electronis, deux ères technologiques se distinguent. La première est l'ère de la technologie électrotechnique, qui requiert un travail sur des mécanismes de précision, comparables à de l'horlogerie (cinq anciens horlogers figurent d'ailleurs dans les effectifs). La seconde est l'ère de la technologie numérique, qui nécessite des compétences totalement différentes de celles de la première technologie. Les jeunes salariés sont formés à la dernière mais méconnaissent la première. Progressivement, les opérations de maintenance des appareils fonctionnant avec la technologie électrotechnique diminuent avec la montée en puissance de technologies plus récentes. Dans le cas présent, l'entreprise a choisi de former de jeunes salariés à l'intervention sur des mécanismes d'horlogerie, une ancienne technologie, et de les faire travailler sous le tutorat des anciens afin de mieux transférer les compétences. L'effort consenti s'explique par la volonté de disposer d'une capacité d'intervention sur d'anciens appareils qui fonctionnent toujours.

\section{La technologie simplifie le travail}

Un troisième facteur vient limiter l'importance des compétences acquises par les salariés les plus anciens. Il est lié à la conception du travail, qui permet de simplifier un processus complexe, notamment en le découpant en unités élémentaires relativement simples. Ce mouvement n'est pas récent, puisqu'il s'agit là d'un principe taylorien mais l'amélioration des capacités de l'automatisation de certaines tâches renforce le processus. Ainsi, il est inutile de disposer d'une expérience de dizaines d'années quand on peut en quelques semaines 
acquérir le savoir-faire nécessaire à la réalisation des tâches essentielles.

L'exemple de Char illustre bien cette situation. L'usine a connu une croissance à la fois de la production de chariots et de son effectif. Alors que 3500 chariots élévateurs par an pour 350 personnes étaient fabriqués en 1984, 25000 chariots par an pour 600 salariés sont assemblés en 2004. En vingt ans, l'entreprise est passée d'un ratio de 10 chariots par personne à un ratio de 41 chariots par personne. Cette augmentation de la productivité, révélatrice d'une intensification du travail qu'on retrouve dans d'autres secteurs d'activité (AskÉNAZY, 2004; Cartron, Gollac, 2003), s'explique essentiellement par la conception de produits plus simples à fabriquer: réduction du nombre d'opérations et préparation d'un assemblage plus facile à réaliser. Chaque renouvellement de chariot est l'occasion de gains de productivité.

Dans la même entreprise, d'autres activités de soudure sont robotisées: dans un caisson fermé, un laser soude les pièces sans que personne n'ait à intervenir. La machine fonctionne sous commandes numériques. Un technicien élabore le programme, qui est ensuite mis en œuvre par des opérateurs. Ces derniers contrôlent la manœuvre, vérifient la profondeur de la soudure et préparent les opérations suivantes en parallèle, quand le robot est activé. La robotisation a eu un double effet sur les compétences des salariés. D'un côté, elle soulage de tâches difficiles, qui demandent précision, maîtrise des gestes et des outils. De l'autre, elle ôte à l'opérateur un savoir-faire manuel en simplifiant certes son travail, mais en lui demandant plus de suivi que d'exécution et plus de préparation, et nécessitant finalement d'autres manipulations manuelles de produits.

\section{Le développement de la polyvalence favorise la transmission des savoir-faire avant la fin de carrière}

Un dernier élément vient pondérer la vision érigeant l'expérience comme un capital essentiel de la personne. Il a trait paradoxalement aux politiques de développement de la polyvalence ou de la polycompétence. En effet, le chemin qui conduit à la polycompétence est fait de formalisation et de diffusion des savoir-faire, ce qui destitue de fait les salariés de compétences rares, quelle que soit leur ancienneté.

Ainsi chez Davia, la DRH a cherché à repérer les personnes clés dans l'établissement, disposant d'un savoir-faire unique. Le départ de ces salariés jugés stratégiques est alors préparé environ six mois avant leur départ. Or, il s'est avéré que peu de personnes détenaient des compétences uniques, compte tenu de la politique de développement des compétences. Dans l'entreprise, l'adoption de la norme ISO 9001 version 2002 a requis la formalisation des compétences, ainsi que l'élaboration de procé- dures écrites et de fiches d'instruction. La normalisation et les pratiques apparentées au Knowledge Management(2) sont deux pratiques managériales contemporaines dont les conséquences ont pour point commun de formaliser et de transmettre les procédures de travail.

Paradoxalement, les politiques qui visent à élargir ou enrichir le travail et à organiser des évolutions de carrière en interne pourraient également avoir pour conséquence de dévaloriser les compétences de quelques-uns, parce qu'elles deviennent les compétences de tous. À cet égard, les modes de gestion que nous avons recensés dans les entreprises étudiées, c'est-à-dire le tutorat, la conservation de la mémoire et le développement de la polycompétence, qu'on pourrait qualifier de vertueux, ont également pour conséquence paradoxale de soustraire aux seniors leurs compétences jugées stratégiques. Finalement, le cas de Davia nous incite à penser que plus ces modes de gestion sont systématisés et intégrés à l'organisation du travail, moins il est nécessaire d'entreprendre le transfert des compétences des seniors.

Reste à savoir si le travail formalisé suffit à résumer le travail réel, si l'expérience accumulée à des postes différents de l'entreprise équivaut à la somme des expériences découpées, et si au-delà des compétences purement liées à l'exécution du travail, le départ des plus anciens ne révélera pas une dimension invisible du travail, peu valorisée jusqu'à présent, par exemple l'appropriation de règles de fonctionnement, l'intégration de gestes de sécurité, la connaissance des dysfonctionnements rares et des façons d'y réagir.

\section{Au-delà de l'apparente prise en compte « rationnelle» de l'expérience}

Un certain nombre d'arguments « rationnels » est avancé pour expliquer la faible prise en compte de l'expérience des seniors. Nous les avons exposés plus haut. Ces explications ne nous suffisent pas pour comprendre ce phénomène, non pas que nous doutions de la sincérité des discours, mais la conception de l'objet "expérience professionnelle» qui y est exposée repose sur un point de vue partiel: d'abord, le discours sur l'expérience est centré sur l'individu, le senior en l'occurrence, en occultant le plus souvent la relation individu-organisation. Ensuite, l'expérience est considérée sous le seul angle des savoir-faire techniques, négligeant les compétences sociales et cognitives. La vision de

(2) Knowledge Management: concept englobant diverses pratiques de formalisation des procédures et manières de travailler afin de diffuser plus facilement les savoirs. 
l'expérience des seniors exprime enfin une vision simplifiée des seniors eux-mêmes, vision confortée par une tradition de l'éviction. Nous détaillons ces points dans la seconde partie de l'article.

Alors que nous avons cherché à rassembler les points communs des discours dans la première partie de l'article, nous examinons à présent les points communs de leurs postulats, de ce qui apparaît comme une évidence telle qu'elle ne nécessite plus d'être questionnée. Nous ne fouillons plus les verbatim mais leurs non-dits.

\section{Une conception de l'expérience centrée sur l'individu}

Nous avons dit précédemment que tous les individus ne tiraient pas parti de l'expérience avec un profit identique, à l'exemple du jeune soudeur jugé plus performant après quelques semaines que d'autres soudeurs disposant d'une ancienneté plus longue. Cependant, le processus de «transformation de soi» par l'expérience (AsTIER, ibid.) mérite d'être replacé dans un contexte organisationnel et ainsi sorti d'une perspective purement individuelle.

L'activation d'un savoir-faire s'inscrit d'abord dans une trajectoire particulière: pour les plus jeunes, le plus souvent en intérim ou en contrats de courte durée, obtenir des résultats élevés est un moyen de se faire reconnaître pour éventuellement bénéficier de contrats plus stables, alors que les plus anciens ne sont plus dans cette préoccupation. Le questionnement de ses propres gestes pour améliorer son efficacité entre dans cette trajectoire. De plus, la réflexivité n'est pas qu'une compétence personnelle; elle peut être encouragée par des mesures organisationnelles. Par exemple, certains dispositifs participatifs de type cercle de qualité peuvent amener des équipes à entreprendre une démarche réflexive qui ne viendrait pas spontanément (SAINSAULIEU, 1987). À cet égard, le travail qui est demandé lors de la VAE requiert réflexivité puisqu'il demande aux candidats de faire de leur expérience professionnelle un objet d'analyse. Dans une certaine mesure, il leur permet de prendre conscience que le travail les a transformés et de sortir la compétence acquise de la singularité de la situation professionnelle pour passer de compétences tacites à des compétences formalisées, de «savoirs investis dans des situations historiques» (SchWARTZ, 2004) à des savoirs plus facilement transférables (Bellini, 2006; VergNies, 2004; Magnier, Werthe, 2001). Enfin, le contenu des emplois fournit lui-même des occasions d'acquérir plus ou moins d'expérience. Un travail répétitif apporte non seulement peu d'occasions d'apprendre dans le travail mais aussi peu d'occasions de réfléchir ou de remettre en cause les façons de travailler alors que la mobilité fonctionnelle les favorise. Le processus d'acquisition d'expérience renvoie ici au couple emploi-individu (VINCENS, ibid.; Rose in Vergnies, ibid.). La notion d'orga- nisation apprenante illustre bien ce processus en énonçant que l'organisation du travail peut placer l'individu dans des situations favorables à de nouveaux apprentissages. Selon Zarifian, (1999), trois conditions doivent être réunies pour qu'il soit présent: occasions d'apprentissage par la confrontation à des évènements (vs opérations répétitives), transfert des apprentissages dans des situations de communication et existence de raisons sérieuses pour effectuer ces apprentissages.

La faculté à tirer parti de l'expérience n'est donc pas qu'un attribut individuel. Trajectoires, dispositifs encourageant la réflexivité, situations de travail sont autant d'éléments qui montrent que l'expérience professionnelle mérite d'être étudiée, non plus en se focalisant sur l'individu, senior ou non, mais en ouvrant la perspective à la relation individuorganisation.

\section{Une vision tronquée de l'expérience}

À travers les propos recueillis lors de nos entretiens, apparaît une conception dominante de l'expérience, à savoir une somme de savoir-faire acquis à un poste de travail. C'est précisément ce savoir-faire qui n'est pas valorisé pour les raisons indiquées plus haut.

Cependant, cette conception nous semble tronquée, puisqu'elle néglige une partie des acquis de l'expérience moins objectivable parce qu'invisible et que de nombreux auteurs mettent en évidence, à quelques nuances près. GRASSER et RoSE (2000) estiment que l'expérience comporte une composante de savoir-faire et une composante de connaissances spécifiques et tacites du processus de production. Aubret et Gilbert (2003) distinguent dans les pratiques, «ce qui est observable et objectivables», et leurs effets qui peuvent être «aussi bien de l'ordre de la conscience spontanée, du ressenti, que le produit d'une analyse ou d'une interprétation ». Rouby et Thomas (2004) opèrent une distinction similaire, entre les «connaissances codifiables » et les «connaissances tacites», moins aisément codifiables. On retrouve ces deux dimensions chez Mallet et VerniÈres (1981), pour qui l'expérience est constituée d'une dimension technique et d'une dimension sociale. La première dimension recouvre la maîtrise des savoir-faire acquis dans l'exercice d'un emploi, alors que la seconde porte sur l'intégration dans le milieu, l'appropriation du langage, des réflexes professionnels ou des codes informels de comportement.

Nous parlions nous-mêmes à propos de Davia d'une dimension invisible de l'expérience, qui pouvait se traduire par l'appropriation de règles de fonctionnement, l'intégration à la mémoire collective de certaines règles comme les règles de sécurité, ce qu'on appelle parfois la culture sécurité.. Nous pouvons également évoquer l'intégration à un réseau interne informel, qui peut être une source de 
gain de temps considérable (savoir qui fait quoi et qui a fait quoi dans le passé) ou encore la connaissance de l'historique de l'entreprise (procédés, tests antérieurs...). Il nous semble que l'expérience, telle que nos interlocuteurs nous en ont parlé, relevait essentiellement de la dimension objectivable et bien peu de l'invisible.

\section{Le processus subjectif d'évaluation de l'expérience professionnelle}

Que 1'on parle de la dimension technique de l'expérience ou de sa dimension sociale et cognitive, ses représentations dominantes sont chaque fois préjudiciables aux seniors. En effet, d'un côté, l'expérience technique est valorisable quand les savoir-faire qu'elle a permis de maîtriser sont d'actualité, mais, son intérêt est fréquemment remis en cause en raison des évolutions technologiques, nous l'avons détaillé plus haut. De l'autre côté, les représentations de l'expérience sociale et cognitive des seniors leur est défavorable. "Les anciens ont pris des habitudes qu'il est difficile de changer» (Char), «ils ne sont plus motivés par leur travail» (Cendrillon), «ils ont du mal à comprendre qu'on ne peut plus faire comme avant » (Satelinergie). Ces phrases sont reprises fréquemment par les DRH. La littérature sur le sujet confirme que des comportements professionnels comme rapidité de réaction, capacité d'adaptation, aptitudes à changer sont attribués aux jeunes et sont estimés déficients chez les anciens, à tort ou à raison. Ainsi on leur confère une rigidité et un manque de créativité qui ont pour conséquence de les écarter des formations et des postes à responsabilité (VolKoff, Molinié, Jolivet, 2000). Ces représentations négatives à l'encontre des salariés les plus âgés de l'entreprise sont d'autant plus marquées que les responsables des ressources humaines sont jeunes. Ainsi, lors du choix d'un candidat pour un poste, les recruteurs déclarent préférer des jeunes puisqu' «ils s'adaptent mieux aux nouvelles technologies et au changement, car ils sont plus polyvalents mais aussi plus dynamiques et plus motivés que les salariés âgés ». (Minni, Topiol, 2004). L'absence d'outils d'évaluation des acquis cognitifs et sociaux de l'expérience laisse la place à la généralisation, à la simplification des traits de personnalité, à la projection de présupposés sur les seniors, bref aux mécanismes psychosociaux du stéréotype.

Pourtant, des études sur la productivité des salariés âgés ne confirment pas ces représentations négatives. Ainsi, selon Gautié (2004), la productivité des salariés restés en emploi ne semble pas décliner, en moyenne, avec l'âge. Le déclin des performances dépend en fait d'une combinaison complexe de variables (individus et situations) qu'on ne peut résumer au seul facteur âge. De plus, les salariés de plus de 55 ans sont les plus représentés dans les anciennes entreprises et sont souvent affectés à des postes qui ne sont pas dotés des équi- pements récents, permettant la meilleure productivité (Aubert, CRÉPOn, 2004). Enfin, les stratégies d'économie d'énergie ou de compensation que les seniors mettent en place sont peu prises en compte (Volkoff, Molinié, Jolivet, ibid.), ce qui constitue pourtant un acquis de l'expérience.

L'expérience des seniors ferait partie du capital social de l'entreprise, mais ce capital ne fait l'objet d'aucune mesure ou estimation, alors que les coûts salariaux le sont avec précision. Faute de pouvoir être valorisée financièrement, l'expérience n'a finalement qu'un coût, croissant avec l'ancienneté. D'où les effets de noria recherchés dans le remplacement d'un salarié à forte ancienneté par un jeune moins expérimenté, mais moins cher.

L'examen des pratiques montre que la préférence donnée aux connaissances des jeunes salariés par rapport à l'expérience des anciens est largement partagée, contrairement aux discours bienveillants sur les compétences des anciens. On peut y voir bien plus que l'expression d'un stéréotype, la traduction d'un modèle de société qui valorise les traits de la jeunesse en lui associant des traits positifs (dynamisme, enthousiasme, activisme). Le "jeunisme », qui consiste à "faire de la jeunesse une valeur en soi »(COMTE-SPONVILLE, cité par Aubret et Gilbert, 2003, p. 114) met en lumière les qualités de la jeunesse et laisse dans l'ombre les apports des salariés vieillissants. En se préoccupant de rajeunir leurs effectifs, les pratiques de GRH entrent dans le processus de reproduction du modèle: elles fabriquent autant qu'elles sont le reflet de ce modèle de société.

\section{L'empreinte des modes de gestion par l'éviction}

Le recours aux mesures d'âge a laissé de profondes traces dans les entreprises, tout à la fois chez les salariés et les DRH. On les trouve mises en place chez Flying System, Prévoir, Flaco, Davia et Satelinergie. De manière générale, c'est un moyen «habituel» de gestion des seniors (ANGLARET, Massin, 2002). Les salariés anciens y voient une solution confortable de fin de carrière. Selon le DRH de Flying System, les mesures d'âge sont considérées comme un droit par de nombreux salariés. Pour les DRH, elles constituent un moyen de rajeunir l'effectif sans dégâts sociaux, voire en obtenant une bonne image sociale. Enfin, les représentants du personnel y trouvent la possibilité de négocier des solutions satisfaisantes pour les plus anciens et de valoriser ainsi leur rôle de défense des salariés. Finalement, chacun a trouvé un intérêt à recourir à ce type de mesures.

Indépendamment des conséquences financières de ces dispositifs sur la collectivité, les mesures d'âge ont longtemps exonéré les organisations d'une réflexion sur la place des seniors dans les organisations et, partant, de mise en place d'une réelle gestion 
des seniors (Pollpot-Rocaboy, 1996). Elles sont devenues, surtout pour les grandes entreprises, une norme managériale autant que sociale qui entérine le critère d'âge comme motif de départ. Au bout du compte, l'utilisation massive des mesures d'âge a fortement contribué à percevoir dans un salarié âgé avant tout le membre d'une classe d'âge et bien peu le détenteur d'une expérience professionnelle.

On aurait tort de considérer aujourd'hui le recours aux mesures d'âge comme une sale manie, alors qu'elle a longtemps été présentée et utilisée comme une pratique vertueuse de gestion des seniors. Avec l'extinction de ces dispositifs et l'allongement de la durée des carrières, ce sont non seulement de nouvelles pratiques qu'il faut construire mais aussi la présence des seniors dans l'entreprise qu'il faut repenser. On peut penser que les pratiques d'autofinancement des mesures d'âge resteront circonscrites à quelques grandes entreprises et resteront inabordables pour la majorité des PME, d'où l'intérêt d'une meilleure prise en compte des acquis de l'expérience des seniors.
Point de salut pour les seniors? Plusieurs facteurs se conjuguent pour dévaloriser leur expérience. L'évolution technologique rend obsolète une partie de leurs acquis et l'ancienneté n'est pas considérée comme un gage d'acquisition de savoir-faire. En outre, les pratiques contemporaines de management, en cherchant à formaliser, normaliser et diffuser les savoir-faire ou en développant la polyvalence, dépossèdent les salariés les plus expérimentés de leurs connaissances.

Pourtant, les arguments dits rationnels légitiment mais n'expliquent que partiellement la faible prise en compte des seniors. Ils traduisent à la fois une conception tronquée de l'expérience professionnelle et une vision simplifiée de ce que sont les salariés âgés. Explorer les fondements des conceptions de l'expérience n'est pas une démarche vétilleuse mais ouvre des perspectives, car la mise à jour de ce que peut apporter l'expérience, considérée dans toutes ses dimensions (technique mais aussi sociale et cognitive), est probablement une piste à approfondir pour maintenir ou développer l'emploi des seniors. L'enjeu organisationnel sous-jacent est de créer l'environnement propice à une certaine réflexivité, soit par des outils de management appropriés (bilan de compétences, participation à l'amélioration du poste ou de l'unité de travail), soit par le développement d'organisations apprenantes. Les salariés gagneraient alors à faire preuve des acquis de leur expérience, qu'ils soient seniors ou non. 


\section{Bibliographie}

Anglaret D., Massin M. (2002), «Les préretraites : un outil important de la gestion des âges dans les entreprises », Premières synthèses, $\mathrm{n}^{\circ}$ 45-1, DARES.

AskénAzy P. (2004), Les désordres du travail. Enquête sur le nouveau productivisme, Paris, collection «La république des idées », Le Seuil.

Astier P., (2004), «Parler d'expérience», Formation emploi, $\mathrm{n}^{\circ}$ 88, octobre-décembre, Marseille, pp. 33-42.

Aubert P., CréPON B. (2004), «La productivité des salariés âgés: Une tentative d'estimation», Économie et Statistiques, $\mathrm{n}^{\mathrm{o}} 368$, avril.

Aubret J., Gilbert P. (2003), Valorisation et validation de l'expérience professionnelle, collection «Topos», Paris, Dunod.

Bellini S. (2006), «Valider l'expérience du travail. Exercice d'anthropologie appliqué à un jury de VAE», Actes du XVIIe congrès de l'AGRH, Reims, novembre.

Bellini S., Duyck J-Y., Laval F., Renaud E., Vauclin S. (2006), «Une typologie des pratiques de gestion des seniors », Revue Management \& avenir, $\mathrm{n}^{0}$ 7, janvier.

Cartron D., Gollac M. (2003), «Intensité et conditions de travail», 4 pages du CEE, $\mathrm{n}^{\circ} 58$.

Diallo M. D., Clot Y. (2003), «L'exploration de l'expérience dans l'analyse de l'activité: problèmes de méthode», Orientation Scolaire et Professionnelle, 32, no 2, pp. 203-217.

FALCOZ C. (2004), Parcours professionnel et employabilité des 50 ans et plus: représentations, pratiques de gestion et enjeux socio-économiques, rapport de recherche, site de l'ANACT, août.

GAUTIÉ J. (2004), «Les travailleurs âgés face à l'emploi», Éonomie et Statistique no 368 , avril.

Giodarno Y. (2003), Conduire un projet de recherche. Une perspective qualitative, Éditions Management et Société.

Grasser B., Rose J. (2000), «L'expérience professionnelle, son acquisition et ses liens à la formation», Formation Emploi, no 71, juillet-septembre, Marseille, pp. 5-19.

Grasser B., Rose J. (2001), «Usage de l'expérience professionnelle et performance collective», Formation Emploi, no 73, Marseille, pp. 5-17.
LinHART D. (1991), Le torticolis de l'autruche ou l'éternelle modernisation des entreprises, Paris, La découverte.

MAllet L., Vernières M. (1981), «L'expérience professionnelle dans les modes de gestion de la main-d'œuvre», Travail et Emploi, no 9, juillet-septembre, pp. 65-74.

Magnier J., Werthe C., (2001), «L'expérience revisitée à l'occasion de la validation des acquis professionnels », Formation Emploi, $\mathrm{n}^{0}$ 75, Marseille, pp. 29-41.

Martin de Holan P., Philips N., (2004), "Remembrance of Things Past? The dynamics of Organizationnal Forgeting", Management Science, vol. 50, $\mathrm{n}^{0} 11$, November, pp. 1603-1613.

Minni C., Topiol A. (2002), «Les entreprises se préoccupent peu du vieillissement démographique», Premières synthèses, $\mathrm{n}^{\circ} 15.1$, DARES.

Minni C., Topiol A. (2004), "Les entreprises face au vieillissement de leurs effectifs », Économie et Statistique $n^{\circ} 368$, avril.

Poilpot-Rocaboy G. (1996), «Performance de la gestion des emplois: éviction ou rétention des salariés vieillissants?», Actes du VIIe congrès de l'AGRH, pp. 423436.

Rouby, E., Thomas, C., (2004), «La codification des compétences organisationnelles: l'épreuve des faits », Revue française de gestion, vol. 30, nº 149, pp. 51-68.

Sainsaulieu R. (1987), Sociologie de l'organisation et de l'entreprise, Paris, Dalloz.

SChWARTZ Y. (2004), «L'expérience est-elle formatrice?», Éducation permanente no 158/2004-1.

Vergnies J.-F. (2004), «La validation des acquis de l'expérience, nouveaux enjeux, nouveaux débats. Entretien avec Labruyère C. et Rose J.», Formation Emploi, $\mathrm{n}^{\circ}$ 88, Marseille.

VinCEns J. (2001), «Définir l'expérience professionnelle», Travail et Emploi no 85, janvier, 21-34.

Volkoff S., Molinié, A. F., Jolivet, A. (2000), Efficaces à tout âge - vieillissement démographique et activités de travail, Centre d'études de l'emploi, dossier 16.

ZARIFIAN P. (1999), Objectif compétence, Paris, Liaisons. 
